\title{
BMJ Open 'Only twice a year': a qualitative exploration of 6-month antiretroviral treatment refills in adherence clubs for people living with HIV in Khayelitsha, South Africa
}

Claire Marriott Keene (D) , ${ }^{1}$ Nompumelelo Zokufa, ${ }^{1}$ Emilie C Venables, ${ }^{2,3}$ Lynne Wilkinson, ${ }^{4,5}$ Risa Hoffman, ${ }^{6}$ Tali Cassidy, ${ }^{1,7}$ Leigh Snyman, ${ }^{1}$ Anna Grimsrud, ${ }^{4}$ Jacqueline Voget, ${ }^{8}$ Erin von der Heyden, ${ }^{8}$ Siphokazi Zide-Ndzungu, ${ }^{8}$ Vinayak Bhardwaj, ${ }^{1}$ Petros Isaakidis ${ }^{2}$

To cite: Keene CM, Zokufa N, Venables EC, et al. 'Only twice a year': a qualitative exploration of 6-month antiretroviral treatment refills in adherence clubs for people living with HIV in Khayelitsha, South Africa. BMJ Open 2020;10:e037545. doi:10.1136/ bmjopen-2020-037545

- Prepublication history for this paper is available online. To view these files, please visit the journal online (http://dx.doi org/10.1136/bmjopen-2020037545).

Received 07 February 2020 Revised 28 April 2020 Accepted 15 May 2020
Check for updates

(C) Author(s) (or their employer(s)) 2020. Re-use permitted under CC BY-NC. No commercial re-use. See rights and permissions. Published by BMJ.

For numbered affiliations see end of article.

Correspondence to Dr Claire Marriott Keene; clairekeene@gmail.com

\section{ABSTRACT}

Objective Longer intervals between routine clinic visits and medication refills are part of patient-centred, differentiated service delivery (DSD). They have been shown to improve patient outcomes as well as optimise health services-vital as 'universal test-and-treat' targets increase numbers of HIV patients on antiretroviral treatment (ART). This qualitative study explored patient, healthcare worker and key informant experiences and perceptions of extending ART refills to 6 months in adherence clubs in Khayelitsha, South Africa.

Design and setting In-depth interviews were conducted in isiXhosa with purposively selected patients and in English with healthcare workers and key informants. All transcripts were audio-recorded, transcribed and translated to English, manually coded and thematically analysed. The participants had been involved in a randomised controlled trial evaluating multi-month ART dispensing in adherence clubs, comparing 6-month and 2-month refills.

Participants Twenty-three patients, seven healthcare workers and six key informants.

Results Patients found that 6-month refills increased convenience and reduced unintended disclosure. Contrary to key informant concerns about patients' responsibility to manage larger quantities of ART, patients receiving 6-month refills were highly motivated and did not face challenges transporting, storing or adhering to treatment. All participant groups suggested that strict eligibility criteria were necessary for patients to realise the benefits of extended dispensing intervals. Six-month refills were felt to increase health system efficiency, but there were concerns about whether the existing drug supply system could adapt to 6-month refills on a larger scale.

Conclusions Patients, healthcare workers and key informants found 6-month refills within adherence clubs acceptable and beneficial, but concerns were raised about the reliability of the supply chain to manage extended multi-month dispensing. Stepwise, slow expansion could avoid overstressing supply and allow time for the health system to adapt, permitting 6-month ART refills to enhance

\section{Strengths and limitations of this study}

- This study is one of the first to qualitatively evaluate perceptions of antiretroviral treatment refills longer than 3 months.

- The experiences of patients in both the standard of care 2-month refill clubs and the 6-month refill clubs are reported, as well as those of staff and key informants.

- Interviewer positionality was reviewed throughout the study by holding regular debriefings with the study team to clarify findings and how the researcher's background may have affected them.

- Interviewers were Médecins Sans Frontières employees and supported the parent trial, which may have influenced how open the participants felt they could be in their interviews.

- Recruitment of particular patient groups was challenging, therefore men, patients under 30 years old and those not retained in care might have been under-represented.

current DSD options to be more efficient and patientcentred within current health system constraints.

\section{INTRODUCTION}

Improved and simplified antiretroviral therapy (ART) means that HIV can be managed as a chronic disease, but requires the health system to adapt to respond to the changing needs of patients on lifelong treatment. Service delivery of HIV care has evolved from a 'one-size fits all' approach to respond to the diverse need of people living with HIV in resource limited settings. Patientcentred, differentiated service delivery (DSD) models for clinically stable ART patients, such as adherence clubs, have proven successful in 
both improving patient outcomes and optimising health services to provide quality care to the increasing numbers of patients on ART. ${ }^{12}$ Multi-month ART dispensing has been endorsed by the WHO to further reduce the burden of care on both patients and the health system ${ }^{3}$ and scale-up of 6-month refills has been accelerated as part of the United States President's Emergency Plan For AIDS Relief (PEPFAR) 2019 country operational plan. ${ }^{4}$

Evidence from multiple studies and a 2016 systematic review suggest that extending refill and visit intervals is safe for patients, with equivalent or better outcomes (retention in care, viral load (VL) suppression, morbidity and mortality) ${ }^{5-9}$ In addition, longer intervals have been shown to improve the clinical efficiency, reduce waiting time and improve satisfaction with services. ${ }^{10}$ They also increase the number of patients that healthcare workers (HCW) can manage, ${ }^{11}$ essential in the era of "universal test and treat' as larger numbers of patients are initiated on ART. However, there is limited qualitative evidence on the impact of extended dispensing intervals to 6 months, particularly with patient and HCW perspectives. ${ }^{1}$

In Khayelitsha, South Africa, Médecins Sans Frontières (MSF) successfully piloted the adherence club model for clinically stable patients, where a group of patients receive their ART refills together through a lay facilitator, who also provides education. ${ }^{12-14}$ The model has now been endorsed in policy and scaled up throughout the country. ${ }^{13}$ MSF implemented a randomised controlled trial to compare extending ART refill intervals from 2 to 6 months among existing adherence club patients. ${ }^{15}$ Preliminary results show non-inferiority in terms of 12-month retention (97\% compared with 98\%) and VL suppression (98\% compared with $97 \%$ ). ${ }^{9}$

This qualitative study explored patient, HCW and key informant experiences and perceptions of extending ART refills to 6 months within the adherence club model.

\section{METHODS}

\section{Study design}

This is a descriptive qualitative study including in-depth interviews (IDIs) with patients, HCWs and key informants.

\section{Setting}

\section{Khayelitsha, South Africa}

Khayelitsha is a large, peri-urban informal settlement outside of Cape Town, South Africa, home to a population of at least 500000 people. It has the highest HIV prevalence in the Western Cape province ${ }^{16}$ with approximately 47000 adults on ART in 2019, of which $30 \%$ of are men and $42 \%$ receive ART in adherence clubs. It has one of the longest standing and largest treatment programmes in South Africa. ${ }^{16}$

The population is extremely mobile, with many people moving between the informal settlement and a neighbouring province. Additionally, high rates of unemployment, violence, mental health issues and substance abuse contribute to poor engagement with health services. ${ }^{16-19}$
Randomised controlled trial of extended ART refills within adherence clubs

A cluster-randomised controlled non-inferiority trial extending ART refills from 2 to 6 months within existing adherence clubs was conducted from 2017 to 2019 at Site B Community Health Centre, a large provincial, primary care clinic in Khayelitsha. ${ }^{15}$ The study was conducted within routine adherence club conditions. The intervention has been described in detail elsewhere ${ }^{15}$ and is summarised in table 1 below.

This qualitative study was conducted to explore perceptions, concerns, benefits and burdens related to extending the ART refill length within this trial.

\section{Study population, sampling and recruitment}

Patients in the trial database were categorised by, age, gender and whether they were retained in their club at the time of enrolment into the qualitative study. This was done to ensure that a balance of perspectives was obtained and that we recorded the views of participants for whom the model of care was successful at facilitating adherence (those who were retained in club care and therefore also virologically suppressed (<400 copies/mL) at last blood draw), and those who were removed from the adherence club as it was no longer the appropriate model of care for them (removed due to VL failure, missing a club appointment or another condition making them ineligible for club care, such as tuberculosis or pregnancy).

Patients from the 6-month arm were interviewed to understand their experiences, and a small sample of patients from the 2-month arm was recruited to differentiate between experiences of the club model itself and the longer refill interval. Eligible patients for the qualitative study were purposively sampled and recruited telephonically by an MSF counsellor.

All HCWs involved with the care of patients in the trial were invited to participate, and included Department of Health doctors, nurses and pharmacists, not-for-profit club facilitators (lay counsellors), an MSF nurse and an MSF pharmacy assistant. HCWs were individually invited to participate after introductions from the clinic's operational manager and direct managers for MSF staff. Follow-up to organise the logistics and discuss participation was conducted over email or text message.

Key informants included individuals from the Department of Health involved in the management of ART programmes and pharmacy processes, and who had influence over the adoption of extending ART refill duration for clinically stable ART patients in the Western Cape. Key informants were selected using snowball sampling, beginning with those identified by the research team, and were contacted by email.

\section{Data collection}

IDI guides were piloted with MSF staff not involved with the trial and revised. Patients were interviewed in isiXhosa by a bilingual research assistant using guides developed in 
Table 1 Procedures and comparison of the standard of care and intervention arms in the parent randomised controlled trial of extended ART refills ${ }^{12-15}$

\begin{tabular}{|c|c|c|}
\hline & $\begin{array}{l}\text { Standard of care: } \\
\text { 2-month refill adherence clubs }\end{array}$ & $\begin{array}{l}\text { Intervention: } \\
\text { 6-month refill adherence clubs }\end{array}$ \\
\hline Eligibility & \multicolumn{2}{|c|}{$\begin{array}{l}\text { Eligibility for adherence club care } \\
\text { Adults over } 18 \text { years } \\
\text { On the same ART regimen for at least } 6 \text { months } \\
\text { Undetectable VL } \\
\text { No current tuberculosis or pregnancy or condition requiring regular clinical } \\
\text { All participants were previously enrolled in an adherence club care at Site B } \\
\text { Community Health Centre }\end{array}$} \\
\hline Adherence club visits & $\begin{array}{l}\text { Frequency: } \\
\text { Two monthly (four visits receiving } \\
\text { 2-month ART refills and one visit } \\
\text { receiving 4-month ART refills over the } \\
\text { December holiday period) (five per year) }\end{array}$ & $\begin{array}{l}\text { Frequency: } \\
\text { Six monthly (two per year) }\end{array}$ \\
\hline \multirow{3}{*}{$\begin{array}{l}\text { Routine bloods (VL and for clinical review, } \\
\text { depending on the regimen) }\end{array}$} & Frequency: Twelve monthly & \\
\hline & Provider: Managed by a nurse & \\
\hline & $\begin{array}{l}\text { Location: At the facility, part of the } \\
\text { adherence club visit }\end{array}$ & $\begin{array}{l}\text { Location: At the facility, an additional } \\
\text { individual visit, scheduled } 1 \text { month before } \\
\text { the adherence club visit }\end{array}$ \\
\hline \multirow[t]{3}{*}{ Clinical consultations } & \multicolumn{2}{|l|}{ Frequency: Twelve monthly } \\
\hline & \multicolumn{2}{|l|}{ Provider: Managed by a nurse } \\
\hline & \multicolumn{2}{|c|}{ Location: At the facility (part of the adherence club visit) } \\
\hline Grace period & \multicolumn{2}{|c|}{ Patients can collect medication up to 5 days after their scheduled appointment } \\
\hline
\end{tabular}

ART, antiretroviral treatment; VL, viral load.

English. Interviews were conducted either at the patient's home or MSF office (based on the patient's preference).

HCW and key informants were interviewed in English by the Principal Investigator (PI). Interviews were conducted in a private office, a clinician's room or a boardroom.

All IDIs were conducted between June and November 2019 and were audio-recorded. Data collection continued until saturation was reached. The PI and research assistant both work for MSF and held fortnightly debriefings to reflect on the impact of their position on the data collection and analysis, and discuss emerging themes from the interviews (including differences and similarities between the participant groups), to allow continued exploration of emerging themes.

\section{Data analysis}

Interviews were each transcribed once and all isiXhosa interviews were translated into English. Transcripts, including the translation of the data, were reviewed by the original interviewer and compared with handwritten field notes to check the quality and validate the data.

All transcripts were manually coded and thematically analysed by the PI, using a network approach to develop primary themes then group them into organising and overarching themes, as described by Attride-Stirling. ${ }^{20}$ Selected transcripts were coded by the research assistant and a co-investigator and were then discussed among the research team to agree on the coding and analysis framework.

\section{Patient and public involvement}

We did not directly include patient and public involvement in this study, but the research question was developed by a team who work closely with patients and the community involved. Patients have been invited to be a part of the dissemination of results and the advocacy efforts that stem from this study and the parent randomised controlled trial.

\section{Informed consent}

All participants gave written consent in English or isiXhosa. The specific roles of key informants and HCW's organisations are anonymised to protect their identity. Patients were reimbursed in cash for their time and transport costs and the amount was clarified during the recruitment and consent process. 


\begin{tabular}{|c|c|c|c|c|c|}
\hline Study number & Age & Sex & Study number & Age & Sex \\
\hline \multicolumn{6}{|l|}{ Patients $(P)$} \\
\hline P6M_1 & 42 & $\mathrm{~F}$ & P2M_1 & 38 & $\mathrm{~F}$ \\
\hline P6M_2 & 45 & $\mathrm{~F}$ & P2M_2 & 58 & $\mathrm{~F}$ \\
\hline P6M_4 & 40 & $\mathrm{~F}$ & P2M_4 & 49 & $\mathrm{~F}$ \\
\hline P6M_5 & 42 & $M$ & P2M_5 & 36 & $\mathrm{~F}$ \\
\hline P6M_6 & 36 & $\mathrm{~F}$ & P2M_6 & 35 & $\mathrm{~F}$ \\
\hline P6M_7 & 59 & $\mathrm{~F}$ & P2M_7 & 54 & $\mathrm{~F}$ \\
\hline P6M_11 & 52 & $\mathrm{~F}$ & & & \\
\hline P6M_12 & 41 & $\mathrm{~F}$ & & & \\
\hline P6M_13 & 40 & $\mathrm{~F}$ & & & \\
\hline P6M_14 & 39 & $\mathrm{~F}$ & & & \\
\hline P6M_15 & 30 & $\mathrm{~F}$ & & & \\
\hline P6M_16 & 47 & $\mathrm{~F}$ & & & \\
\hline \multicolumn{3}{|c|}{ Healthcare worker (HCW) } & \multicolumn{3}{|c|}{ Key informants (KI) } \\
\hline HCW_1 & 59 & $\mathrm{~F}$ & KI_1 & Not collected & M \\
\hline HCW_2 & 59 & $\mathrm{~F}$ & KI_2 & & $\mathrm{F}$ \\
\hline
\end{tabular}

\section{RESULTS}

\section{Demographics}

A total of 36 IDIs were conducted (ranging in length from 19 min to 1 hour), including 23 patients (16 in 6-month and 7 in 2-month ART refill clubs), 7 HCWs and 6 key informants. Table 2 summarises the demographics and roles of the interviewees.

\section{Themes}

Patient, HCW and key informant perspectives were combined in six overarching themes that emerged from the data: three relating to the impact of extending ART refills to 6 months on the patients, two relating to the impact on the health system and one overlapping theme exploring eligibility (see figure 1 ).

\section{Impact on patients}

Extending ART refills to 6 months within the club model influenced patients and their care in several ways, including (1) the convenience of ART engagement, (2) unintended disclosure and (3) their responsibility and motivation.

\section{Convenience}

Patients, HCWs and key informants agreed that extending ART refills to 6 months decreased obstacles to accessing facilities, reducing the burden of frequent clinic visits and reducing travel costs for patients

It made a big difference. I am now saving all the R20s (transport money) (P6M_8)

Patients described time as very valuable and a major benefit of 6-month refills is that 'it saves a lot of time' (P6M_10). The time saved by not attending the club visit is used to go to work, look for employment, child care, household chores, shopping and addressing challenges that arise in their lives.

It helps them a lot because these patients now have got the time to look after themselves (HCW_2)

Patients noted that increasing the time between visits allows for extended travel: for work or to address issues where family live outside of the province. 


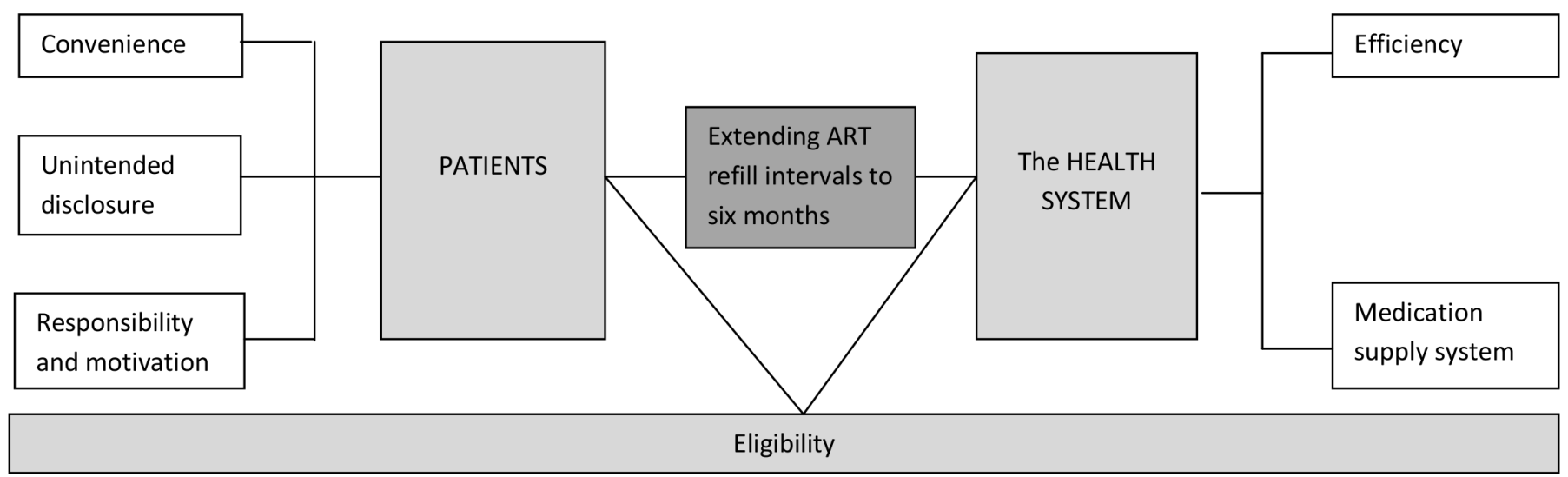

Figure 1 Overarching themes related to the impact of the model on patients and the health system. ART, antiretroviral treatment.

It changed my life so much because I know that I have my pills for 6 months. For example, we go out, we attend funerals, we have problems in our homes (rural areas) so now I can stay the whole month or 3 months at home. I couldn't do that when I was in 2 months because I had to rush and come back for my appointment and if you missed it you must start afresh. So now it saves time and it saves everything (P6M_10)

Six-month ART refills were described by HCWs as á huge help for patients who are working' (HCW_4) as 'it's not easy to get jobs so if they found a job they don't want to lose it' (HCW_7). Employment was noted by patients as a significant barrier to accessing the health system, either because going to work prevented them from attending their appointment or travel took them out of the area. Attending an ART appointment rather than work was described as stressful by patients due to the loss of income and the risk of loss of employment if work was missed:

I did not like 2 months as I was going to the clinic all the time. I could see that I would lose my job because some bosses don't like you telling them that you are HIV positive. Even when I go to the clinic I don't want the paper (sick note). [Six-month clubs] are not difficult like the 2 months. In the 2 -month club you have to go to the clinic, and that can lead you to be fired by your bosses because they will ask 'what's wrong with this person who goes to the clinic every 2 months?' (P6M_5)

Patients felt they 'had to lie in the 2 month (club)... and come up with new excuses' (P6M_10) because they feared annoying their employer by asking for frequent days off and were worried about disclosing their HIV status to employers that they did not trust.

Longer ART refills allowed patients more time to organise and plan their lives, and reduced the stress of trying to balance their life and ART commitments. It also provided patients with a sense of security to manage stock-outs or uncertain medication supply.
Six months was good for me, because when you have to do something you are always anxious. Six months is a lot, you have peace of mind, and you know you have your pills in front of you. Even if something happens and you are told the pills are finished, I will know I have mine: only those that gets 2 months supply will be affected (P6M_2)

\section{Unintended disclosure}

HCWs and key informants were concerned that larger numbers of ART bottles would result in unintended disclosure when transporting the medication and storing it at home. Patients were more concerned about the noise that the large number of bottles made.

My only problem is the noise that they make. They are in these containers and there is a lot of them... so they make noise and I cannot go everywhere with them. I was worried when a friend of mine called me to come to her place. I was in a taxi, so I had to get off and go to her. That did not make me feel good because I was carrying my pills. I am refusing to let go of my bag, because I am like 'leave my bag alone dude!' That stressed me. I knew that even if I wanted to stay longer, I had to quickly go because now I can't even go outside and leave my bag, and when I am touching my bag, it is making a noise and you can see that its pills. She was asking what was in my bag, what are these pills for? But I can't tell her. (P6M_5)

Patients noted that less frequent visits reduced the risk of disclosure at work, because 'the day you mention the word HIV, is the day you lose your job' (P2M_4). Patients in both 6-month and 2-month clubs were not greatly concerned about disclosure at home as most had disclosed to their immediate family and asked them for support with adherence. Some patients described being 'afraid to be seen in the clinic' (P6M_1). Patients, HCWs and key informants felt that 6-month refills provided patients with more privacy and could help to reduce 
unintended disclosure as community members were less likely to assume infrequent visits were for ART.

I would say there is a change because the frequent visits to the library to get medication meant that even people who didn't know your status could tell you were part of the people who went there, which would raise questions. Now they won't be able to see that because you won't be going as frequently (P6M_9)

\section{Responsibility and motivation}

Key informants were concerned that if patients were provided with larger quantities of medication they would use it irresponsibly, adherence would worsen and seeking care when ill would be delayed until a scheduled appointment. They were also concerned about suboptimal storage of medication, that patients might share or sell medication or that it would be stolen.

There were cases where they told us it was stolen. We know for a fact that the drugs do get used for other purposes as well and we know of instances where people were collecting ARV's and selling them. But who knows, most of them had lost them, or they were stolen, or they don't know why they're finished (KI_5)

The experiences reported by patients did not support these concerns; patients reported many mechanisms to remind them to take their treatment daily and attend appointments, including setting phone reminders, using calendars, timing their pills with popular television shows and asking family members to remind them. Patients in clubs valued their health and their treatment and reported being highly self-motivated in both the 6 -month and 2-month clubs.

I am taking my treatment well, this is my health and my life, and I don't have another life. My life depends on me (P6M_10)

What is important in my life is my life (P2M_7)

Patients reported making use of the grace period or would rebook if they missed an appointment, and reported that they either had, or would, visit health services if they became sick in between visits or lost their medication

You don't have to wait the whole 6 month until your date for you to come to the clinic... I would go to the clinic like everyone else and tell the doctor I am in the club but I am sick at that time (P6M_9)

Patients reported having a specific place to store their medication that was cool, dark and safe from theft and children's access. Most said that they do not share their medication, because staff have instructed them not to, other people are not using the same regimens, they worry about running out of pills and they value the treatment for their own use.
The pills are calculated for me not her, she must go to the clinic to take hers... I can't give anyone my pills. Even my siblings know these are mine not for everyone (P6M_2)

Two participants (P6M_5 and P6M_13) reported that they had shared medication, but only with their spouses under exceptional circumstances and only if they were on the same regimen. They also had plans to mitigate the impact on their adherence: sharing a limited amount, requiring the medication to be returned or accompanying the recipient to the clinic for their refill.

Theft of medication (in the facility, in transit or at home) was not reported by any of the participants and was not a major concern. Patients had heard about those who smoke and sell them' (P6M_10) but this was unsubstantiated by specific examples. Participants felt pills were lost as collateral in the theft of a bag rather than being the target of theft, thus patients took action to mitigate theft of their belongings.

I take a taxi, because there are pickpockets, and come straight home (P6M_15)

In addition, patients and HCWs reported that the benefits of 6-month ART refills actually increased patient adherence further.

We are very trusted with the 6 months... we are given enough time to look after ourselves, that is where our confidence is built (P6M_3)

\section{Impact on the health system}

From the HCW and key informant perspective, the main benefit for the system was increased efficiency and the main concern was the impact on the medication supply system. Some participants noted that despite the benefits of longer refills, there were still significant challenges and for some the non-inferior trial outcomes were not enough to justify the risks and additional investment required to implement longer duration in ART dispensing. Ultimately, there was cautious optimism about 6-month refills, with key informants acknowledging the benefits while warning of the concerns and practical challenges described below.

\section{Efficiency}

From the perspectives of the HCWs and key informants, one of the main benefits of 6-month ART refills for the health system was the 'efficiency gains' and the ability to 'service more people with the same number of staff' (KI_5). It was reported that the need to do this was driven by pressure from the 'universal test and treat' guidelines:

They are coming every 2 months or every 6 months: that's a third that you have cut... That's huge... Now that we are trying to start everyone who gets tested on ARVs, the numbers we are looking at are going to be immense. (HCW_4) 
The HCW workload for each club visit was described as remaining the same or slightly increasing (particularly for pharmacists) but increasing the refill interval within the adherence club model resulted in reduced workload overall, particularly for club facilitators and data clerks:

Personally it has helped me a lot because it reduces the workload... So that gives me a little relief. It's not that I am relieved from them, but I'm relieved from the workload... So you prepare your registers, you prepare the medication and you must be earlier than usual when you are going to do the club. It's more preparation if the patient is going to be...doing a clinical visit and taking bloods. But now I don't have that big preparation. They are giving me a gap. (HCW_2)

HCWs reported that the additional time created by seeing patients less frequently was used to manage other activities that were previously neglected or more stressful due to high patient volumes, including administration and management of ad hoc problems in the clinic.

Patients in the 6-month clubs perceived there to be an increase in peer and club facilitator support, despite concerns from key informants that the reduced visit frequency would diminish support. Paradoxically the decreased visit frequency in the 6-month model increased the quality of the support because patients could take an entire day off work instead of rushing through the session in order to attend to other competing tasks for the day, as they had been doing when in 2-month clubs. Patients and HCW also described patients enjoying the reunion with peers when they returned for the club visit.

When they come back, they come back with that excitement. 'We have been away from the clinic, how are you?' They are happy! They are now enjoying the club. (HCW_2)

\section{Medication supply system}

A major concern raised by key informants and HCWs was the current medication supply system's capacity to adapt to 6-month refills. They expressed concern regarding the smaller facilities with limited storage space that would require more frequent deliveries from the depot. Key informants felt that the supply system was already 'on a knife's edge' (KI_1) and planning was dependent on factors outside local control, including manufacturers, national contracts and demand from other provinces.

You need good stocks to be able to hand that out... But with what happens at national level, sometimes our systems are not good enough and that could create a real disaster. (KI_4)

While acknowledging that for the ART programme itself, it's been very rare to have stock outs' (KI_4) compared with other chronic disease treatment, it was suggested that the impact of stock-outs for patients receiving 6-month ART refills could detrimentally impact supply by creating peaks in the demand for medication. There was concern over the industrial amounts of medicine' that would be in the community rather than within the health system's control.

It's a lot of medicine to be sitting in people's homes ... So it's a lot of money tied up in people's homes where you don't have access to it as you would in the health system... I mean that's a lot of medicine that's going to be sitting out in communities. (KI_2)

'Ultimately the drugs are for the community' (KI_3) and slow and careful expansion of longer refills was suggested as a way to avoid 'absolute disaster' (KI_4), allowing time for planning and problem solving. This would reduce stress on the supply chain and the risk of overwhelming the system.

\section{Eligibility}

There was consensus across key informants, HCWs and patients from the 6-month and 2-month clubs that if the existing club model were adapted to provide 6-month refills, it would not be appropriate for everyone and would benefit a 'select small group of patients' (KI_2). There was consistency across interviewees in suggesting strict criteria with evidence of long-term adherence, such as multiple suppressed VL results. It was proposed that newly initiated patients need experience on ART to develop maturity, build up understanding and knowledge and learn to solve challenges. Both HCWs and patients suggested that stable ART patients eligible for the adherence club model should start in the 2-month clubs and 'graduate' to 6-month refills when they had proved themselves.

You cannot skip to the 6 months if no one knows how you took the 2 months. They have to see that you are taking the 2-month supply first to show that you are taking it well. If you are not taking the 2 month well, don't even bother going to the 6 months. If you are someone who puts their health before anything, you can take the 6 months supply. I would advise them to go to the clinic and start with the 2 months and see if you manage and if you do, you can get on the 6 months. (P2M_4)

It was also recommended that patients should 'earn' the reward of 6-month refills, as one patient described:

We also started there [2-month refills]. We were standing in those queues. It was not easy for us: we worked for that 6 months. They must also work for it so that they can get to where we are. They must start with the 2 months: we started from the bottom and we climbed our way up until we were alright. (P6M_8)

Six-month ART refills were viewed by HCWs and patients as a reward for good adherence, with patients viewed as 'VIPs' who feel like champions' (HCW_1). Six-month refills motivated and empowered patients to 
be adherent and take control of their treatment, with the possibility of losing the benefits ensuring adherence.

They took their treatment more seriously because they loved being in the 6 month (club) and you tell them if the viral load increases unfortunately, I won't be able to keep you in the 6-month club. They took their medication because they wanted to be in the 6 -month arm. They continue to be quite motivated, to take treatment and take control. (HCW_5)

\section{DISCUSSION}

This study is one of the first to qualitatively evaluate perceptions of ART refills longer than 3 months, ${ }^{21}$ and explore patient, HCW and key informant experiences of 6-month refills for clinically stable HIV patients in adherence clubs. Six-month refills were recently trialled by MSF and the Western Cape Department of Health in South Africa, with non-inferior patient outcomes to the standard of care clubs receiving 2-month ART refills. ${ }^{9}$

All participants agreed that 6-month refills were a motivation for adherence and that the main benefit was the increase in free time and convenience, even in addition to the time-saving benefits described previously for the club model itself. ${ }^{2}$ Longer refills also gave patients 'peace of mind' and a sense of control in managing their treatment, despite the uncertainties in their lives and the health system, such as stock outs.

Our data suggest that patients did not struggle with adherence, storage or safety of their medication, contrary to key informant concerns. Patients valued their health and their treatment, but also valued their life responsibilities, such as work and family. The frequency of ART visits was a significant stress and in their experience of 2-month refills, patients reported finding ways around their visits that did not jeopardise other obligations. Patients live in complicated worlds that require navigation of competing priorities, ${ }^{22}$ and if a choice has to be made, life commitments may be prioritised at the expense of ART. Six-month refills helped to overcome this tension by reducing the impact of many previously described barriers to engagement with the health system, such as travel, stigma, inconvenience of appointments, changes to routine and being busy preventing attendance. ${ }^{23}$ As the health system's focus shifts to mechanisms of retaining patients in lifelong care, it is increasingly necessary for DSD to put patient's preferences at the centre of service design. ${ }^{122}$ Longer refill intervals support patients to incorporate ART into their daily life and can enhance the convenience of current DSD models.

In addition to patient benefits, 6-month refills were felt to increase the health system's efficiency, a welldocumented benefit of $\mathrm{DSD}^{2}$ and ascribed to reduced visit frequency in previous studies. ${ }^{11}$ This is an important consideration in the era of 'test and treat', where growing numbers of people starting ART has not been met with an increase in the number of HCWs to support this expansion. A majority of patients on ART are expected to be eligible for DSD models and this is likely to have a significant impact on DSD infrastructure. ${ }^{24}$ Current DSD options need further evolution to make even more efficient use of resources, in order to cope with the growing ART cohort.

A significant concern for HCWs and key informants was whether the existing drug supply system could adapt to provide 6-month ART refills at scale. The precariousness of the supply chain would both limit the feasibility of implementation and be worsened by the additional stress longer ART refills would place on the system. These concerns highlight the critical importance of DSD optimisation, health system planning and close monitoring of phased implementation at national and provincial levels to ensure feasibility and long-term sustainability.

Patients (including those receiving 2-month refills), HCWs and key informants alike felt that extended refills were only suitable for a select group of patients, who needed to both prove themselves (with evidence of longterm adherence such as multiple suppressed VL results) and earn the benefits by 'doing their time' with shorter refill intervals. While eligibility criteria are important to ensure that DSDs are implemented appropriately, meeting these criteria does not necessarily result in successful outcomes. ${ }^{24}$ The need for strict eligibility criteria needs to be balanced with the patient-centred benefits 6-month refills could offer to clinically stable patients.

There are several limitations to this study that bring into question the transferability of the findings to other settings. It was challenging to recruit men, patients under 30 years old and those lost from clinic care at the time of recruitment for this qualitative study. These groups accounted for a small proportion of the original study population (23\%, $4.5 \%$ and $5 \%$, respectively) and were also particularly difficult to contact during the recruitment process. The participants in the randomised controlled trial from which the participants for this qualitative study were drawn, were recruited from existing adherence clubs (eligibility for which demonstrates long-term adherence) questioning the transferability of the findings to settings that do not offer group DSD models or to patients not yet virologically suppressed on ART. However, participants in the 2-month refill clubs were also included to explore whether 6-month refills impacted the experience of those who were already shown to be stable patients.

Some of the benefits described by patients and HCW, such as the 'reward' of being chosen for the 6-month refill clubs, or the special 'VIP' treatment, came from a misunderstanding of the randomisation process (despite multiple rounds of consent) as 'being chosen'. Clubs are already seen as a reward for 'taking treatment well', and pre-existing confusion about 'qualifications' for club eligibility has been previously described in this population, which may have contributed to misunderstandings about the further differentiation to 6-month refills. ${ }^{2}$

Interviewers were MSF employees and supported the parent trial, which could have potentially influenced how 
open the participants felt they could be in their interviews. This positionality was reviewed and discussed in regular debriefings to reduce the impact on the collection and analysis of data. Patients may also have been reluctant to share challenges in case this jeopardised the continuation of the model, however this could also be perceived as their belief in the benefits of the model and their desire to continue in it.

\section{CONCLUSION}

Six-month refills have previously been demonstrated to be safe in terms of patient outcomes, and this qualitative study established that extending ART refill length within the adherence club model was also highly acceptable and valued among patients and HCWs. Key informants were cautiously optimistic about the benefits for selected longterm clinically stable patients, but remained concerned about feasibility of implementation with supply chain posing the biggest challenge.

The evolution of current models is necessitated by the continuing need to increase the number of patients on ART under 'universal test and treat' and also successfully retain them on lifelong treatment. Six-month refills have potential to augment current DSD options to be more efficient and patient-centred, regardless of the model through which ART is provided. Gradual expansion of 6-month ART refills could avoid overstressing supply and allow time for the health system to adapt, potentially facilitating sustainable adherence for large numbers of patients within current resource constraints.

\section{Author affiliations}

${ }^{1}$ Médecins Sans Frontières South Africa, Cape Town, South Africa

${ }^{2}$ Southern Africa Medical Unit, Medecins Sans Frontieres South Africa, Cape Town, South Africa

${ }^{3}$ Division of Social and Behavioural Sciences, School of Public Health and Family Medicine, University of Cape Town, Rondebosch, Western Cape, South Africa ${ }^{4}$ International AIDS Society, Cape Town, South Africa

${ }^{5}$ Centre for Infectious Epidemiology and Research, University of Cape Town, Rondebosch, Western Cape, South Africa

${ }^{6}$ Division of Infectious Disease, David Geffen School of Medicine, University of California Los Angeles, Los Angeles, California, USA

${ }^{7}$ Division of Public Health Medicine, School of Public Health and Family Medicine, University of Cape Town, Rondebosch, Western Cape, South Africa

${ }^{8}$ Western Cape Department of Health, Cape Town, South Africa

Acknowledgements We would like to thank all the interviewees for giving up the time to share their experiences and perceptions with us. We are very grateful to Xoliswa Nxiba and Keitu Lebelo for their efforts to trace the participants who were no longer retained in care, so that we could represent their perspectives and to Zodwa Mgengwana-Mbikaza for assisting with transcription. We would like to thank the MSF staff past and present who worked tirelessly on the 6 months study and Ubuntu Clinic (Site B CHC) and the Khayelitsha-Eastern Subdistrict Department of Health for piloting the 6-month model, and for taking it over to test its feasibility without NPO support. We would also like to thank MSF LuxOR and the SORT-IT course organisers for providing the funding and mentorship to complete this study, and all the SORT-IT participants for their thoughtful comments in the development and write up of this manuscript.

Contributors Conceptualisation: CMK, PI, ECV. Data curation: CMK, NZ. Formal analysis: CMK, PI, NZ, ECV. Funding acquisition: MSF SORT IT course. Methodology: CMK, PI. Project administration: CMK, NZ. Writing - original draft: CMK, PI, ECV. Writing - review and editing: LS, JV, LW, TC, RH, EvdH, VB, SZ-N, AG.
Funding The authors have not declared a specific grant for this research from any funding agency in the public, commercial or not-for-profit sectors.

Competing interests This research was conducted through the Structured Operational Research and Training Initiative (SORT IT), a global partnership led by the Special Programme for Research and Training in Tropical Diseases at WHO (WHO/TDR). The model is based on a course developed jointly by the International Union Against Tuberculosis and Lung Disease (The Union) and Médecins Sans Frontières (MSF/Doctors Without Borders). The specific SORT IT programme that resulted in this publication was managed by MSF and the research was undertaken as part of routine MSF activities with MSF Khayelitsha contributing from staff salaries and operational budget.

Patient consent for publication Not required.

Ethics approval This study was approved by the MSF Ethics Review Board (reference 1910), the University of Cape Town's Human Research Ethics Committee (HREC 191/2019) and the Western Cape Provincial Department of Health.

Provenance and peer review Not commissioned; externally peer reviewed.

Data availability statement Data are available upon reasonable request in accordance with MSF's data sharing policy. Requests for access to data should be made to data.sharing@msf.org.

Open access This is an open access article distributed in accordance with the Creative Commons Attribution Non Commercial (CC BY-NC 4.0) license, which permits others to distribute, remix, adapt, build upon this work non-commercially, and license their derivative works on different terms, provided the original work is properly cited, appropriate credit is given, any changes made indicated, and the use is non-commercial. See: http://creativecommons.org/licenses/by-nc/4.0/.

ORCID iD

Claire Marriott Keene http://orcid.org/0000-0002-0875-5884

\section{REFERENCES}

1 Grimsrud A, Barnabas RV, Ehrenkranz P, et al. Evidence for scale up: the differentiated care research agenda. J Int AIDS Soc 2017;20:22024-6.

2 Venables E, Towriss C, Rini Z, et al. Patient experiences of art adherence clubs in Khayelitsha and Gugulethu, Cape town, South Africa: a qualitative study. PLoS One 2019;14:e0218340-18.

3 World Health Organization. Consolidated guidelines on the use of antiretroviral drugs for treating and preventing HIV infection: recommendations for a public health approach. World Heal Organ, 2016: 155.

4 PEPFAR U.S. President's Emergency Fund for AIDS Relief. PEPFAR 2019 Country Operational Plan Guidance for all PEPFAR Countries, 2019.

5 Obua C, Kayiwa J, Waako P, et al. Improving adherence to antiretroviral treatment in Uganda with a low-resource facility-based intervention. Glob Health Action 2014;7:24198-9.

6 Kwarisiima D, Kamya MR, Owaraganise A, et al. High rates of viral suppression in adults and children with high CD4+ counts using a streamlined art delivery model in the search trial in rural Uganda and Kenya. J Int AIDS Soc 2017;20:21673-67.

7 Bekolo CE, Diallo A, Philips M, et al. Six-monthly appointment spacing for clinical visits as a model for retention in HIV care in Conakry-Guinea: a cohort study. BMC Infect Dis 2017;17:1-10.

8 Mutasa-Apollo T, Ford N, Wiens M, et al. Effect of frequency of clinic visits and medication pick-up on antiretroviral treatment outcomes: a systematic literature review and meta-analysis. J Int AIDS Soc 2017;20:21647.

9 Lebelo K, Cassidy T, Grimsrud A, et al. Twelve-month retention and viral load outcomes from a non-inferiority cluster randomized trial extending adherence club ART refill dispensing intervals to 6-monthly. In: IAS conference on HIV science 21-24 July 2019. Mexico City, 2019.

10 Alamo ST, Wagner GJ, Ouma J, et al. Strategies for optimizing clinic efficiency in a community-based antiretroviral treatment programme in Uganda. AIDS Behav 2013;17:274-83.

11 Mesic A, Fontaine J, Aye T, et al. Implications of differentiated care for successful art scale-up in a concentrated HIV epidemic in Yangon, Myanmar. J Int AIDS Soc 2017;20:21644-13.

12 Grimsrud A, Sharp J, Kalombo C, et al. Implementation of community-based adherence clubs for stable antiretroviral therapy patients in Cape town, South Africa. J Int AIDS Soc 2015;18:19984-8. 
13 Wilkinson L, Harley B, Sharp J, et al. Expansion of the adherence Club model for stable antiretroviral therapy patients in the Cape Metro, South Africa 2011-2015. Trop Med Int Health 2016;21:743-9.

14 Western Cape Department of Health HAST. Guidelines for art clubs, 2015.

15 Wilkinson L, Grimsrud A, Hayes H, et al. Research protocol - section C exploring 6-month dispensing intervals for adherence clubs - a cluster randomized study, 2016.

16 Stinson K, Goemaere E, Coetzee D, et al. Cohort profile: the Khayelitsha antiretroviral programme, Cape town, South Africa. Int $J$ Epidemiol 2017;46:e21.

17 Harker N, Kader R, Myers B, et al. Substance abuse trends in the Western Cape: a review of studies conducted since 2000, 2008: 1-54.

18 Jacob N. Mental illness in the Western Cape, 2015: 1-35.

19 Western Cape Government. The harms and risks of alcohol in Khayelitsha department of community safety, 2015.
20 Attride-Stirling J. Thematic networks: an analytic tool for qualitative research. Qual Res 2001;1:385-405.

21 International AIDS Society. Facility-based individual: summary of published evidence, 2019. Available: http://www.differentiatedcare. org/Resources/Summary-of-published-evidence/Facility-basedindividual [Accessed 31 Dec 2019].

22 Eshun-Wilson I, Rohwer A, Hendricks L, et al. Being HIV positive and staying on antiretroviral therapy in Africa: a qualitative systematic review and theoretical model. PLoS One 2019;14:e0210408.

23 Shubber Z, Mills EJ, Nachega JB, et al. Patient-Reported barriers to adherence to antiretroviral therapy: a systematic review and metaanalysis. PLoS Med 2016;13:e1002183-14.

24 Hoffman RM, Balakasi K, Bardon AR, et al. Eligibility for differentiated models of HIV treatment service delivery: an estimate from Malawi and Zambia. AIDS 2020;34:475-9. 